\title{
Cervical Spine CT Using Spectral Shaping: Can It Be a Solution to Overcome Artifacts in the Lower Cervical
} Spinal Region?

\author{
Su Min Lee, $M D^{1}$, Hye Jung Choo, MD, $\mathrm{PhD}^{1}$, Sun Joo Lee, MD, $\mathrm{PhD}^{1}$, Sung-Kwan Kim, $\mathrm{MD}^{1}$, \\ In Sook Lee, MD, PhD' ${ }^{2}$, Dong Wook Kim, MD, PhD ${ }^{1}$, Jin Wook Baek, MD $^{1}$, Young Jin Heo, MD \\ ${ }^{1}$ Department of Radiology, Inje University Busan Paik Hospital, Busan, Korea; ${ }^{2}$ Department of Radiology, Biomedical Research Institute, Pusan \\ National University Hospital, Busan, Korea
}

Objective: To investigate the image quality, radiation dose, and intermodality agreement of cervical spine CT using spectral shaping at $140 \mathrm{kVp}$ by a tin filter (Sn140-kVp) in comparison with those of conventional CT at $120 \mathrm{kVp}$.

Materials and Methods: Patients who had undergone cervical spine CT with Sn140-kVp ( $n=58)$ and conventional $120 \mathrm{kVp}(\mathrm{n}=$ 49) were included. Qualitative image quality was analyzed using a 5-point Likert scale. Quantitative image quality was assessed by measuring the noise and attenuation within the central spinal canals at C3/4, C6/7, and C7/T1 levels. Radiation doses received by patients were estimated. The intermodality agreement for disc morphology between CT and MRI was assessed at C3/4, C5/6, C6/7, and C7/T1 levels in 75 patients who had undergone cervical spine MRI as well as CT.

Results: Qualitative image quality was significantly superior in Sn140-kVp scans than in the conventional scans $(p<0.001)$. At C7/T1 level, the noise was significantly lower and the decrease in attenuation was significantly less in Sn140-kVp scans, than in the conventional scans $(p<0.001$ ). Radiation doses were significantly reduced in Sn140-kVp scans by $50 \%$ (effective dose: $1.0 \pm 0.1 \mathrm{mSv}$ vs. $2.0 \pm 0.4 \mathrm{mSv} ; p<0.001$ ). Intermodality agreement in the lower cervical spine region tended to be better in Sn140-kVp acquisitions than in the conventional acquisitions.

Conclusion: Cervical spine CT using Sn140-kVp improves image quality of the lower cervical region without increasing the radiation dose. Thus, this protocol can be helpful to overcome the artifacts in the lower cervical spine CT images.

Keywords: Cervical spine; Multidetector computed tomography; Spectral; Metal artifact

\section{INTRODUCTION}

Cervical spine CT is widely used in the evaluation of cervical spinal abnormalities. American College of Radiology has recommended cervical spine $\mathrm{CT}$ as a primary imaging

Received August 3, 2018; accepted after revision November 3, 2018.

This study was supported by the DongKook Life Science. Co., Ltd., Republic of Korea.

Corresponding author: Hye Jung Choo, MD, PhD, Department of Radiology, Inje University Busan Paik Hospital, 75 Bokji-ro, Busanjin-gu, Busan 47392, Korea.

- Tel: (8251) 890-6549 - Fax: (8251) 896-1085

- E-mail: hyejungchoo@gmail.com

This is an Open Access article distributed under the terms of the Creative Commons Attribution Non-Commercial License (https://creativecommons.org/licenses/by-nc/4.0) which permits unrestricted non-commercial use, distribution, and reproduction in any medium, provided the original work is properly cited. modality in suspected spine trauma because of its high sensitivity and specificity to detect fractures (1). Cervical spine CT has been suggested as an alternative modality to MRI even for the diagnosis of a herniated disc or spinal stenosis due to shorter scan time and cost-effectiveness (2). However, the diagnosis of lesions at the lower cervical region on $\mathrm{CT}$ is challenging due to the presence of streaks and dark bands, which are explained as photon starvation or beam-hardening artifacts generated by the shoulder girdles $(3,4)$. The diagnosis of a disc pathology or spinal stenosis is more problematic because the artifacts are usually more severe in the central spinal canals. A simple method to remove the artifacts is by using a high tube current or voltage. However, this is accompanied by a radiation dose penalty. Although there have been trials and technical developments such as shoulder reposition and automatic tube current modulation to solve this issue $(5,6)$, the dark- 
band and streak artifacts in the lower cervical area on CT still remain problematic.

Recently, "spectral shaping" of photon energy spectrum by a tin filter has been introduced. The tin filter, which is added to the standard aluminum bowtie filter between $X$-ray tube and the patients, absorbs low-energy photons that contribute less to the image quality but increase the radiation exposure to patients, and results in an increase in the mean photon energy of the applied radiation (7). Several latest studies demonstrated that the spectral shaping with a tin filter allowed for reduction of the radiation dose, while maintaining the image quality (8-13). Thus, we hypothesized that a high-energy tube voltage with spectral shaping by a tin filter might make it possible to overcome the artifacts in the lower cervical spine area in CT without an increase in the radiation dose.

Therefore, the purpose of this study was to investigate the image quality, radiation dose, and intermodality agreement of cervical spine CT using spectral shaping at 140 $k V p$ by a tin filter (Sn140-kVp) in comparison with those of the conventional cervical spine CT at $120 \mathrm{kVp}(120-\mathrm{kVp})$.

\section{MATERIALS AND METHODS}

This retrospective study was approved and informed consent was waived by the Institutional Review Board.

\section{Study Population}

This study enrolled 136 patients who had undergone non-enhanced cervical spine CT with Sn140-kVp protocol and 105 patients who had undergone conventional 120kVp cervical spine CT between January 2017 and September 2017 in our institution. Among them, the patients with metal devices in the cervical spines $(n=82)$, fracture of the cervical spine $(n=38)$, severe spinal stenosis $(n=11)$, and infection of the cervical spine $(n=3)$ were excluded. Finally, 58 patients ( 31 men and 27 women; mean age, 58 years; age range, 28-83 years) who had undergone CT with Sn140-kVp CT were selected as the study group and 49 (25 men and 24 women; mean age, 57 years; age range, 25-83 years) with conventional CT as the control group. Thirtyseven patients in the study group (23 men, 14 women; mean age, 61 years) and 38 in the control group (21 men, 17 women; mean age, 57 years) underwent cervical spine MRI within three months before or after the cervical spine CT (median interval, 7 days; interval range, $0-90$ days).

\section{CT and MRI Acquisitions}

A third-generation dual-source CT (DSCT) (Somatom Drive, Siemens Healthcare, Forchheim, Germany) was utilized for the Sn140-kVp protocol. This protocol included a dedicated tin filter mounted in front of the $\mathrm{X}$-ray tube, which resulted in the spectral shaping of the polychromatic $X$-ray and increased the mean photon energy by shutting down the low levels of energy. Conventional 120-kVp CT was performed using single-source CT (Somatom Definition AS, Siemens Healthcare). Automatic tube current modulation (CareDose 4D, Siemens Healthcare) was activated in both protocols with a reference tube current-time product of 280 $\mathrm{mAs}$ for Sn140-kVp and $275 \mathrm{mAs}$ for $120-\mathrm{kVp}$. All the images were reformatted into axial and sagittal planes with a 2-mm thickness and the axial planes were reformatted parallel to the orientation of each intervertebral disc. The parameters for each protocol are summarized in Table 1.

The cervical spine MRI was obtained by a 3T MR system (Magnetom Skyra, Siemens Healthcare, Erlangen, Germany) and a 64-channel head-neck coil. The parameters for the cervical spine MRI are summarized in Table 2.

Table 1. Parameters for Cervical Spine CT

\begin{tabular}{lcc}
\hline \multicolumn{1}{c}{ Parameter } & Sn140-kVp & $120-\mathrm{kVp}$ \\
\hline Tube voltage $(\mathrm{kVp})$ & 140 & 120 \\
Reference tube current-time products (mAs) & 280 & 275 \\
Detector configuration $(\mathrm{mm})$ & $128 \times 0.6$ & $128 \times 0.6$ \\
Pitch & 0.6 & 0.6 \\
Rotation time $(\mathrm{sec})$ & 1 & 1 \\
Field of view (mm) & $120 \times 120$ & $120 \times 120$ \\
Reconstruction algorithm & Filtered back projection soft-tissue kernel & Filtered back projection soft-tissue kernel \\
Reformatted section thickness (mm) & 2 & 2 \\
Reformatted section increment (mm) & 2 & 2 \\
\hline
\end{tabular}

Sn140-kVp = cervical spine CT using spectral shaping at $140 \mathrm{kVp}$ by tin filter, $120-\mathrm{kVp}=$ cervical spine CT at $120 \mathrm{kVp}$ 


\section{Image Analysis}

Three radiologists (readers A and B with 11-year and 5-year experiences in musculoskeletal radiology, respectively; reader $\mathrm{C}$ with 3 -year experience in clinical radiology) blinded to the CT protocols and patients' information, reviewed the images.

\section{Body Habitus}

For an arbitrary estimation of the body habitus of the patients, the level and right-to-left (RL) diameter of the shoulder girdles and the anterior-to-posterior (AP) diameter of the neck were evaluated by the reader $\mathrm{C}$ because the body habitus could affect the $\mathrm{CT}$ image quality and radiation dose. The level of the shoulder girdle was recorded as the order of the vertebral body superimposed by the upper margin of the humeral head on the lateral CT scout view. The $\mathrm{RL}$ diameter of the shoulder girdle was measured the level of the superior end of the humerus on an AP CT scout view.
The AP diameter of the neck was measured parallel to the intervertebral disc at C6/7 on the lateral CT scout view (14).

\section{Image Quality}

The image quality was analyzed using both qualitative and quantitative methods. For qualitative image analysis, the three readers independently scored the image quality on the sagittal image plane by using a 5 -point Likert scale (1, non-diagnostic; 2 , limited diagnostic value with substantial image noise and artifacts; 3 , diagnostic, sufficient image quality; 4, diagnostic, good image quality with mild image noise and artifacts; 5, diagnostic, excellent image quality with absent or subtle noise and artifacts) focusing on the inside of the central spinal canal in the lower cervical spinal region including the cervicothoracic junction (13) (Fig. 1). Before the individual review, the readers trained themselves using a training dataset of 10 cases outside the study cohort, which was prepared by the study coordinator. During the

Table 2. Parameters for Cervical Spine MRI

\begin{tabular}{|c|c|c|c|c|}
\hline Parameter & $\begin{array}{l}\text { Sagittal T1-Weighted } \\
\text { Fast Spin-Echo }\end{array}$ & $\begin{array}{l}\text { Axial T1-Weighted } \\
\text { Fast Spin-Echo }\end{array}$ & $\begin{array}{l}\text { Sagittal T2-Weighted } \\
\text { Fast Spin-Echo }\end{array}$ & $\begin{array}{l}\text { Axial T2-Weighted } \\
\text { Fast Spin-Echo }\end{array}$ \\
\hline Repetition time (msec) & 440 & 600 & 4000 & 2950 \\
\hline Echo time (msec) & 11 & 13 & 62 & 108 \\
\hline Matrix size & $512 \times 358$ & $320 \times 256$ & $512 \times 269$ & $384 \times 269$ \\
\hline Slice thickness (mm) & 3 & 3 & 4 & 4 \\
\hline Interslice gap (mm) & 0.3 & 0.3 & 0.5 & 0.5 \\
\hline No. of signals acquired & 2 & 2 & 4 & 2 \\
\hline Imaging time & $2 \min 41 \mathrm{sec}$ & $2 \mathrm{~min} 33 \mathrm{sec}$ & $3 \mathrm{~min} 16 \mathrm{sec}$ & $2 \min 17 \mathrm{sec}$ \\
\hline
\end{tabular}

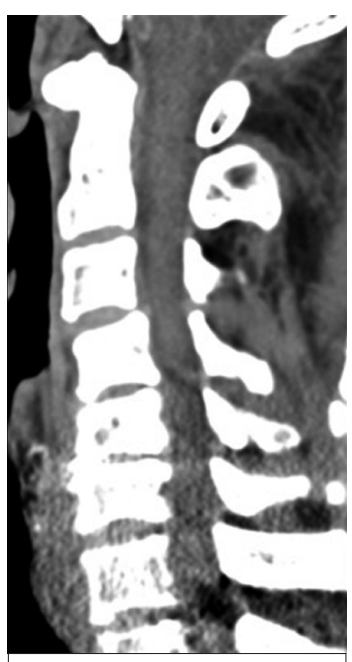

Grade 1

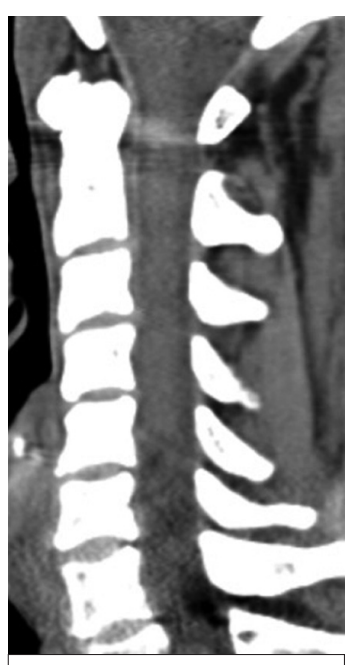

Grade 2

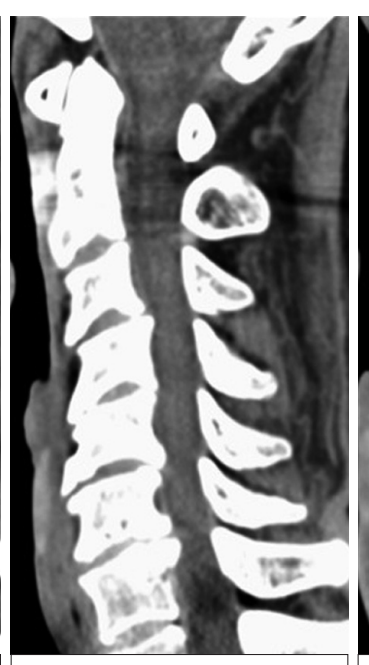

Grade 3
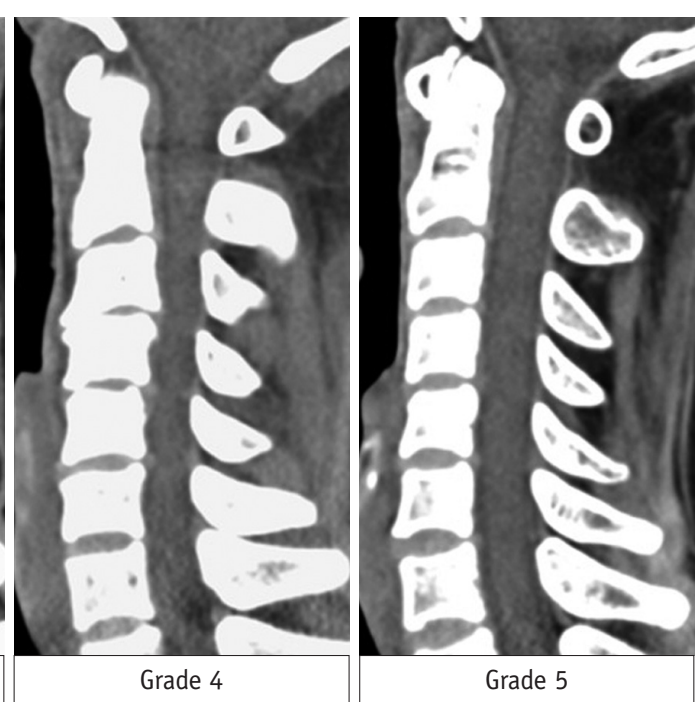

Fig. 1. Grading system for analysis of qualitative image quality. Qualitative image quality was scored on sagittal image plane by using 5-point Likert scale (1, non-diagnostic; 2, limited diagnostic value with substantial image noise and artifacts; 3, diagnostic, sufficient image quality; 4, diagnostic, good image quality with mild image noise and artifacts; 5, diagnostic, excellent image quality with absent or subtle noise and artifacts) focusing on inside of central spinal canal in lower cervical spinal region including cervicothoracic junction. 


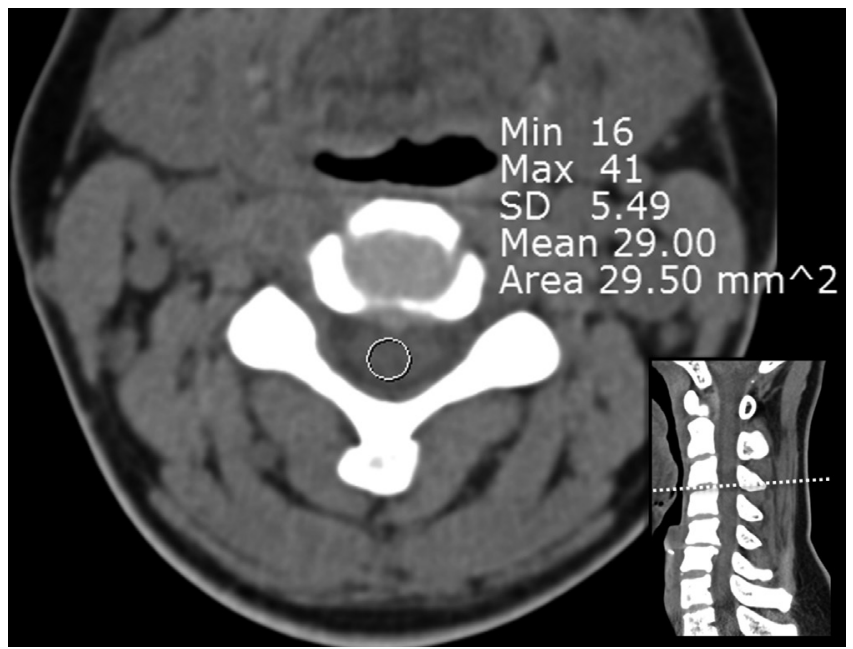

Fig. 2. Measurement for analysis of quantitative image quality. About $30-\mathrm{mm}^{2}$ circular region of interest was placing in center of central spinal canal on axial CT image of intervertebral disc level. To minimize bias, measurements were performed at three sequential slices and averaged. SD = standard deviation

qualitative analysis, all images were displayed with a window level/width set of 45/350 Hounsfield units (HUs).

For quantitative analysis, the CT attenuation values and noises (standard deviation [SD] of the attenuation values) inside the central spinal canals were measured at the intervertebral disc levels of $\mathrm{C} 3 / 4, \mathrm{C} 6 / 7$, and $\mathrm{C} 7 / \mathrm{T} 1$ by the reader $\mathrm{A}$. C3/4 was included for obtaining the baseline data that were not affected by the shoulder girdles. For measurements, an approximately $30-\mathrm{mm}^{2}$ circular region of interest (ROI) was placed in the center of the central spinal canal on the axial image plane (Fig. 2). Additionally, for the evaluation of signal-to-noise ratio (SNR) and contrastto-noise ratio (CNR) of the bones, an approximately 60$\mathrm{mm}^{2}$ circular ROI was placed in the center of the bodies of the $\mathrm{C} 3, \mathrm{C} 6$, and $\mathrm{C} 7$ vertebrae on the mid-sagittal plane. The SNR of the bones was calculated by dividing the average of attenuation values by the SD in the ROI. The CNR between the vertebral body and the central spinal canal was calculated using the following equation (15):

$$
\begin{aligned}
& \mid\left(\text { Attenuation value }_{\text {bone }}-\text { Attenuation value }_{\text {central canal }}\right) \mid / \\
& \sqrt{\left(\mathrm{SD}_{\text {bone }}{ }^{2}+\mathrm{SD}_{\text {central canal }}{ }^{2}\right)}
\end{aligned}
$$

To minimize the bias, all measurements were performed at the three sequential slices and averaged.

\section{Intermodality Agreement for Disc Morphology between CT and MRI}

On the axial and sagittal planes of the cervical spine CT and MRI, the disc morphology was determined into normal disc, bulging, protrusion, and extrusion at the levels of $\mathrm{C} 3 / 4, \mathrm{C} 5 / 6, \mathrm{C} 6 / 7$, and $\mathrm{C} 7 / \mathrm{T} 1$ by two radiologists (readers $\mathrm{A}$ and $B$ ) in consensus (16). The two sets of CT and MRI were reviewed separately.

\section{Radiation Dose}

The radiation exposure to patients was estimated by volume CT dose index (CTDI $\left.I_{\text {vol }}\right)$, dose length product (DLP), and effective dose. The CTDI ${ }_{\text {vol }}$ and DLP were automatically provided by the scanner systems. The effective radiation dose was calculated by multiplying the DLP by the appropriate conversion coefficients, $0.0052 \mathrm{mSv} \times \mathrm{mGy}^{-1} \times \mathrm{cm}^{-1}$ for Sn140-kVp protocol (adult's neck region in $140 \mathrm{kVp}$ ) and $0.0051 \mathrm{mSv} \times \mathrm{mGy}^{-1} \times \mathrm{cm}^{-1}$ for the conventional $120-\mathrm{kVp}$ protocol (adult's neck region in $120 \mathrm{kVp}$ ) (17).

\section{Statistical Analysis}

The normality of the continuous variables was tested by using the Kolmogorov-Smirnov test. The normally distributed variables (age of the patients and diameters of the shoulders and necks) were reported as means \pm SD and compared using the Student's $t$ test. The non-normally distributed continuous variables (noise, attenuation, SNR and CNR of the bone, CTDI ${ }_{\text {vol, }}$ DLP, and effective radiation dose) and the ordinal variable (qualitative image quality) were reported as medians \pm interquartile ranges and analyzed using the Mann-Whitney $U$ test. Fisher's exact test was used for the comparison of the categorical variables (sex and the shoulder level). The relationship between CT radiation doses and diameters of the shoulders and necks was evaluated using the Pearson correlation test.

Interobserver agreement for the qualitative image quality among the three readers was assessed using the weighted kappa (к). Intermodality agreement of the disc morphology between CT and MRI were evaluated in terms of $\kappa$. The degree of agreement ( $\kappa$ value) was categorized as follows: < 0, poor; 0-0.20, slight agreement; 0.21-0.40, fair agreement; $0.41-0.60$, moderate agreement; 0.610.80 , substantial agreement; and $0.81-1$, almost perfect agreement (18).

All statistical analyses were performed using SPSS (SPSS Statistics version 24; IBM Corp., Armonk, NY, USA) and MedCalc (MedCalc Software bvba version 17.11.5, MedCalc Corp., 0stend, Belgium). A two-sided $p$ value of less than 0.05 was considered to indicate a statistically significant difference. 
Table 3. Demographics and Body Habitus of Patients

\begin{tabular}{|c|c|c|c|}
\hline & Sn140-kVp $(n=58)$ & $120-k V p(n=49)$ & $P$ \\
\hline \multicolumn{4}{|l|}{ Demographics } \\
\hline Age (years)* & $58 \pm 14$ & $57 \pm 14$ & 0.557 \\
\hline $\operatorname{Sex}^{\dagger}$ & & & 0.700 \\
\hline Male & $31(53 \%)$ & $25(51 \%)$ & \\
\hline Female & $27(47 \%)$ & $24(49 \%)$ & \\
\hline \multicolumn{4}{|l|}{ Body habitus } \\
\hline Shoulder level ${ }^{\dagger}$ & & & 0.165 \\
\hline C6 & $1(2 \%)$ & $2(4 \%)$ & \\
\hline C7 & $23(40 \%)$ & $16(32 \%)$ & \\
\hline T1 & $29(50 \%)$ & $25(51 \%)$ & \\
\hline T2 & $5(9 \%)$ & $6(12 \%)$ & \\
\hline Shoulder RL diameter $(\mathrm{mm})^{*}$ & $396.0 \pm 33.2$ & $401.5 \pm 32.6$ & 0.419 \\
\hline Neck AP diameter $(\mathrm{mm})^{*}$ & $121.6 \pm 13.1$ & $122.9 \pm 13.4$ & 0.608 \\
\hline
\end{tabular}

${ }^{*}$ Data are means \pm standard deviations, ${ }^{\dagger}$ Data are numbers (percentages). AP $=$ anterior-to-posterior, $\mathrm{RL}=$ right-to-left

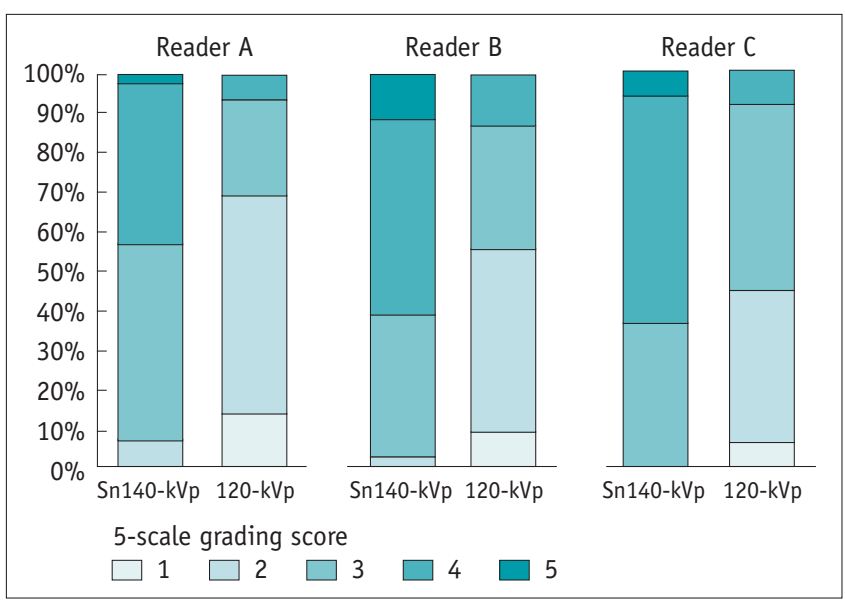

Fig. 3. Column chart for analysis of qualitative image quality. Three readers determined qualitative image quality by using 5 -point Likert scale (1, non-diagnostic; 2, limited diagnostic value with substantial image noise and artifacts; 3 , diagnostic, sufficient image quality; 4, diagnostic, good image quality; 5, diagnostic, excellent image quality). Grading scores are statistically significant higher in cervical spinal CT with Sn140-kVp than 120-kVp by all three readers. Sn140-kVp = cervical spine CT using spectral shaping at $140 \mathrm{kVp}$ by a tin filter, $120-\mathrm{kVp}=$ cervical spine $\mathrm{CT}$ at $120 \mathrm{kVp}$

\section{RESULTS}

\section{Demographics and Body Habitus}

Age and sex of patients, diameters and level of the shoulder girdles, and diameter of the neck were not significantly different between the study and control groups. C7 and T1 were the common levels of the shoulder girdles in both groups (Table 3). In patients who underwent both cervical spine MRI and CT within a 3-month interval, these demographics and body habitus were not significantly different between the two subgroups.

\section{Image Quality}

The scores for the qualitative image quality by all three readers were significantly higher in Sn140-kVp acquisitions than in the conventional acquisitions (median scores: 4 by all readers in Sn140-kVp scans, 3 by readers B and C, and 2 by reader $C$ in conventional scans; $p<0.001$ for all readers) suggesting that the qualitative image quality of $\mathrm{CT}$ with Sn140-kVp acquisitions was significantly superior to that of conventional 120-kVp CT acquisitions (Figs. 3, 4). The scores showed moderate interobserver agreement $(\kappa=0.693$ between readers $A$ and $B ; 0.695$ between readers $A$ and $C$; 0.605 between readers $B$ and $C$ ).

The quantitative image quality was analyzed at the three intervertebral disc levels (Table 4). The attenuation values in the central spinal canals were gradually decreasing, and the noises were gradually increasing from $\mathrm{C} 3 / 4$ to $\mathrm{C} 7 / \mathrm{T} 1$ levels in both groups (Figs. 5, 6). However, the noises at C7/T1 level were significantly lower in Sn140-kVp scans than in the conventional $120-k V p$ scans $(p<0.001)$. The decrease in the attenuation values at $\mathrm{C} 7 / \mathrm{T} 1$ level was also significantly less in Sn140-kVp scans than that in the conventional scans, although the median attenuation value for Sn140-kVp scan was less than $0 \mathrm{HU}$. The noises at $\mathrm{C} 6 / 7$ as well as $\mathrm{C} 3 / 4$ levels were not significantly different between the two groups as these levels were not severely affected by the shoulder girdles, which were mainly at $\mathrm{C} 7$ or $\mathrm{T} 1$ level in the included patients. The attenuation values at C6/7 level were not significantly different between the two groups whereas those at C3/4 level were significantly lower in Sn140-kV scans than in the conventional scans.

The SNR and CNR of the bones were not significantly 


\section{KJR}

different between the Sn140-kV scans and conventional 120-kVp scans in all C3, C6, and C7 levels (Table 5).
Intermodality Agreement for Disc Morphology between CT and MRI

The disc morphology showed almost perfect agreement in
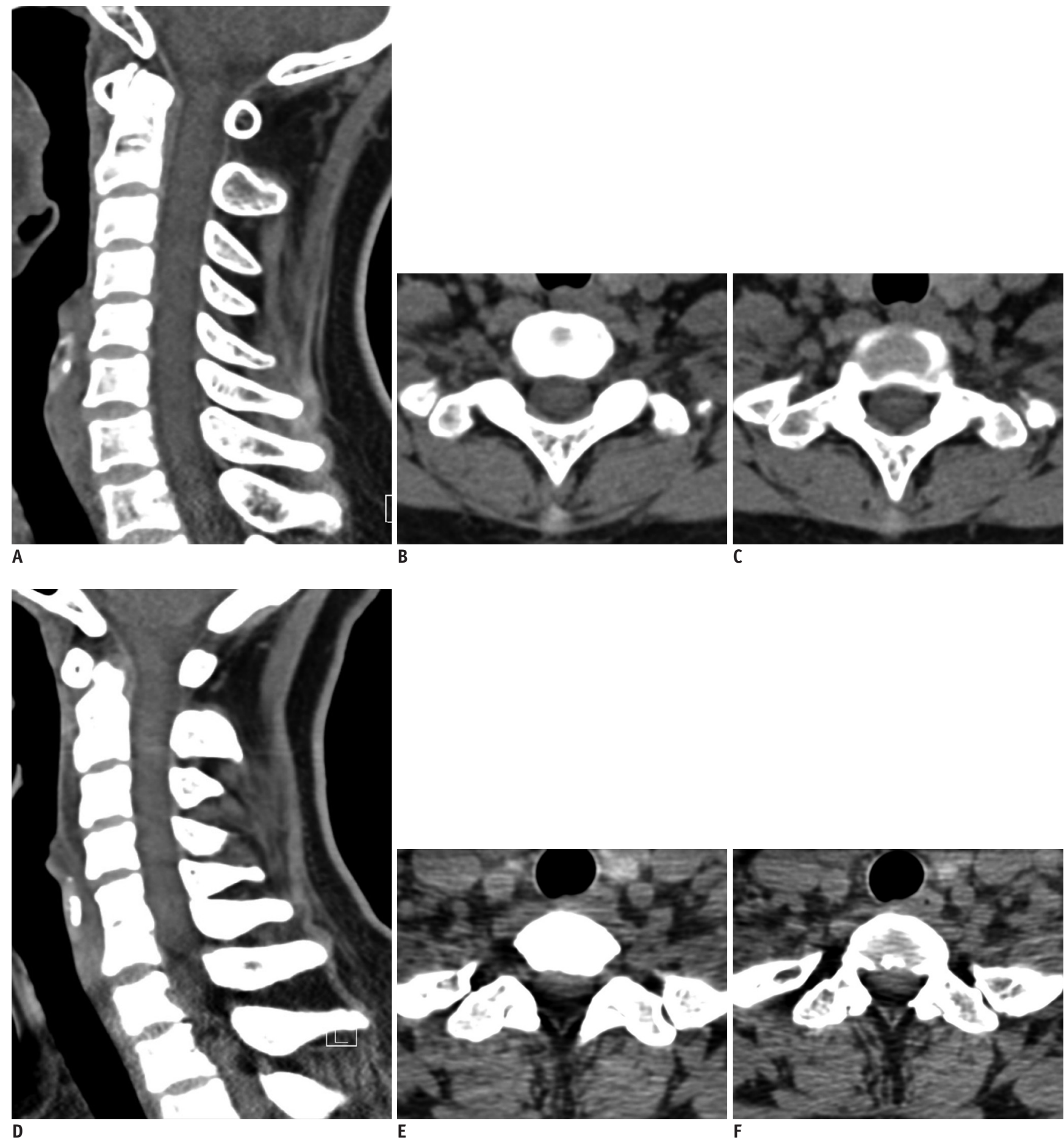

Fig. 4. Comparison of CT images obtained with Sn140-kVp or 120-kVp.

A-C. Cervical spine CT with Sn140-kVp of 49-year-old woman with posterior neck pain (shoulder level, C7; shoulder width, $375 \mathrm{~mm}$; neck diameter, $111 \mathrm{~mm}$ ). D-F. Cervical spine CT with conventional CT with 120-kVp of 43-year-old woman with posterior neck pain (shoulder level, C7; shoulder diameter, $358 \mathrm{~mm}$; neck diameter, $103 \mathrm{~mm}$ ). B, C, E, and $\mathbf{F}$ are two consecutive axial images in level of C7/T1. In spite of similar body habitus of shoulder and neck between two patients, artifacts in lower cervical spinal levels are more severe in conventional scan than Sn140-kVp scan. Congenital blocked vertebrae of $\mathbf{C} 5$ and $\mathbf{C} 6$ is identified in $\mathbf{D}$. 
both Sn140-kV and conventional 120-kVp scans at the C3/4 level where there are no artifacts generated by the shoulder girdles (Table 6). The intermodality agreements for the disc morphology in the lower cervical spinal region were low in both groups; however, the $\kappa$ values were higher for Sn140$k V p$ scans than for conventional scans.

\section{Radiation Dose}

The $\mathrm{CTDI}_{\mathrm{vol}}, \mathrm{DLP}$, and effective dose were significantly reduced in Sn140-kVp scans than in the conventional 120$k V p$ scans $(p<0.001)$; all the medians of the radiation doses for Sn140-kVp scans were about 50\% less than those for the conventional scans (Fig. 7, Table 7).

In the analysis for the relationship between the body habitus and radiation dose, the radiation dose was significantly positively correlated with the diameters of the shoulders and necks in both Sn140-kVp and the conventional acquisitions, although the coefficients tended to be slightly higher in the conventional CT than in Sn140$\mathrm{kVp}$ scans (Table 8). However, the radiation dose among the shoulder levels of $\mathrm{C} 6, \mathrm{C} 7, \mathrm{~T} 1$, and $\mathrm{T} 2$ was not significantly different in both CT scans.

\section{DISCUSSION}

Tin is a non-radioactive and non-volatile material. When it is implemented as a filter in the CT tube, it causes spectral shaping of the polychromatic $X$-ray beam by absorbing the low levels of $\mathrm{X}$-ray energy, which contributes little to the image quality but increases the radiation dose. Hence, a tin filter increases the mean photon energy level and decreases radiation dose exposure to the patients $(7,19)$. Recently, a tin filter was implemented in a high energy tube in dual-energy DSCT, wherein it increased the

Table 4. Quantitative Image Quality of Cervical Spine CT

\begin{tabular}{|c|c|c|c|c|c|c|}
\hline & \multicolumn{3}{|c|}{ Noise (HU) } & \multicolumn{3}{|c|}{ Attenuation (HU) } \\
\hline & Sn140-kVp & $120-k V p$ & $P$ & Sn140-kVp & $120-k V p$ & $P$ \\
\hline $\mathrm{C} 3 / 4$ & $7.4 \pm 1.1$ & $7.9 \pm 2.6$ & 0.902 & $29.7 \pm 3.6$ & $36.7 \pm 5.6$ & $<0.001$ \\
\hline $\mathrm{C} 6 / 7$ & $10.2 \pm 3.2$ & $11.6 \pm 4.8$ & 0.286 & $19.6 \pm 12.1$ & $21.6 \pm 16.4$ & 0.195 \\
\hline $\mathrm{C} 7 / \mathrm{T} 1$ & $15.4 \pm 4.7$ & $22.3 \pm 9.0$ & $<0.001$ & $-2.5 \pm 15.5$ & $-19.9 \pm 22.7$ & $<0.001$ \\
\hline
\end{tabular}

Data are medians \pm interquartile ranges. $\mathrm{HU}=$ Hounsfield units

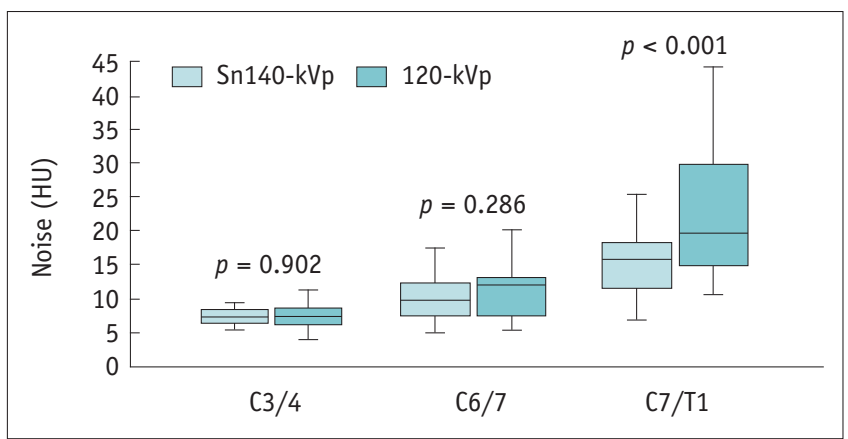

Fig. 5. Box-and-whiskers plots for noise inside central spinal canal. Noise is gradually increased from $\mathrm{C} 3 / 4$ to $\mathrm{C} 7 / \mathrm{T} 1$ in both cervical spine CT scans with Sn140-kVp and 120-kVp. At level of C7/ $\mathrm{T} 1$, noise in Sn140-kVp scans is significantly less than in conventional $120-k V p$ scans. Boxes represent medians and interquartile ranges and whiskers extend to 1.5 interquartile ranges. $\mathrm{HU}=$ Hounsfield units

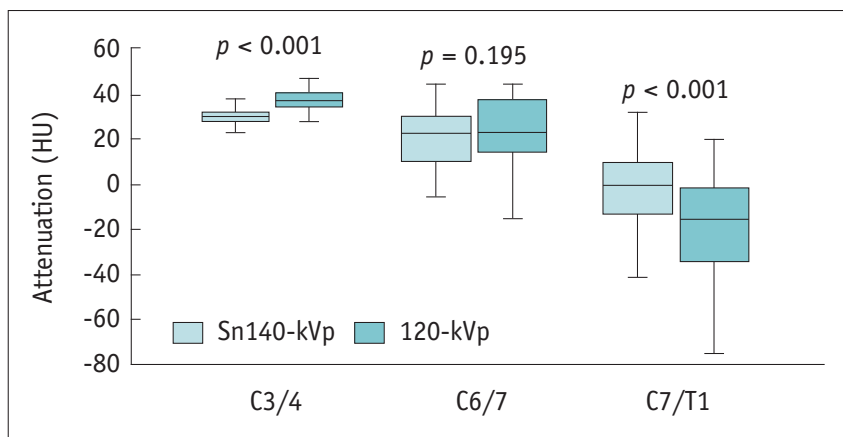

Fig. 6. Box-and-whiskers plots for attenuation values inside central spinal canal. Attenuation gradually decreases from C3/4 to C7/T1 in both cervical spine CT scans with Sn140-kVp and 120-kVp. At level of $\mathrm{C} 7 / \mathrm{T} 1$, attenuation in $\mathrm{Sn} 140-\mathrm{kVp}$ scans significantly less decreases than in $120-k V p$ scans.

Table 5. SNR of Cervical Spinal Bodies and CNR between Spinal Bodies and Central Canal

\begin{tabular}{|c|c|c|c|c|c|c|}
\hline & & SNR $R_{\text {bone }}$ & & & $\mathrm{R}_{\text {bone-central car }}$ & \\
\hline & Sn140-kVp & $120-k V p$ & $P$ & Sn140-kVp & 120-kVp & $P$ \\
\hline $\mathrm{C3}$ & $5.3 \pm 5.3$ & $5.3 \pm 2.1$ & 0.903 & $4.8 \pm 2.2$ & $4.6 \pm 1.9$ & 0.867 \\
\hline C6 & $4.7 \pm 1.9$ & $5.0 \pm 3.4$ & 0.172 & $4.2 \pm 1.9$ & $4.4 \pm 3.2$ & 0.096 \\
\hline $\mathrm{C} 7$ & $5.2 \pm 3.4$ & $5.5 \pm 2.2$ & 0.328 & $4.9 \pm 3.0$ & $5.4 \pm 1.6$ & 0.206 \\
\hline
\end{tabular}

Data are medians \pm interquartile ranges. CNR $=$ contrast-to-noise ratios, SNR = signal-to-noise ratios 
energy separation by minimizing the overlap of high and low $\mathrm{kVp}$ spectra and improved the material discrimination (19-22). In a study using urinary stone samples, DSCT with a tin filter (80/Sn140-kVp) significantly improved the discrimination between the uric acid containing and nonuric acid containing urinary stones as compared with DSCT without a tin filter (80/140-kVp) (20). In a study about

Table 6. Intermodality Agreement for Disc Morphology between Cervical Spine CT and MRI

\begin{tabular}{|c|c|c|}
\hline & \multicolumn{2}{|c|}{ Morphology of Disc } \\
\hline & Sn140-kVp & $120-k V p$ \\
\hline $\mathrm{C} 3 / 4$ & $0.824(0.670-0.978)$ & $0.828(0.668-0.988)$ \\
\hline $\mathrm{C} 5 / 6$ & $0.760(0.562-0.957)$ & $0.655(0.442-0.868)$ \\
\hline $\mathrm{C} 6 / 7$ & $0.658(0.457-0.858)$ & $0.546(0.344-0.748)$ \\
\hline $\mathrm{C} 7 / \mathrm{T} 1$ & $0.547(0.247-0.847)$ & $0.124(-0.162-0.411)$ \\
\hline
\end{tabular}

Data are kappas (95\% confidence intervals).

Table 7. Radiation Dose of Cervical Spine CT

\begin{tabular}{lccc}
\hline & Sn140-kVp & $120-k V p$ & $P$ \\
\hline CTDI $_{\text {vol }}(\mathrm{mGy})$ & $9.8 \pm 1.0$ & $19.8 \pm 1.8$ & $<0.001$ \\
DLP $(\mathrm{mGy} \cdot \mathrm{cm})$ & $195.5 \pm 27.7$ & $395.0 \pm 69.8$ & $<0.001$ \\
Effective dose $(\mathrm{mSv})$ & $1.0 \pm 0.1$ & $2.0 \pm 0.4$ & $<0.001$ \\
\hline
\end{tabular}

Data are medians \pm interquartile ranges. CTDI $_{\text {vol }}=$ volume $\mathrm{CT}$ dose index, $\mathrm{DLP}=$ dose length product gout protocol CT (22), DSCT using a tin filter (80/Sn150$\mathrm{kVp}$ ) had significantly fewer artifacts than DSCT without a tin filter $(80 / 140-k V p)$.

Since the third-generation of DSCT was introduced in 2014, a tin filter is able to be implemented even in a single energy acquisition (23). Chest CT studies using the third generation DSCT with a single-energy mode and tin filter (Sn100-kVp) showed a reduction in radiation dose and equal to superior image quality in comparison with conventional CT without a tin filer (8-10). In the paranasal sinus and temporal bone CT studies, the CT using a tin filter (Sn100$k V p$ and Sn150-kVp, respectively) substantially lowered the radiation exposure to patients, while the image quality was preserved $(11,12)$. Whole body low-dose CT using spectral shaping by a tin filter ( $\mathrm{Sn} 100-\mathrm{kV}$ ) in patients with multiple myeloma also showed a sufficient image quality for the depiction of osteolytic lesions, while reducing the radiation dose (13).

Our study demonstrated similar results as these previous studies using a tin filter: the cervical spine CT with Sn140-kVp allowed a significant radiation dose reduction, preserved the image quality in the upper cervical spinal area, and revealed a significantly better image quality

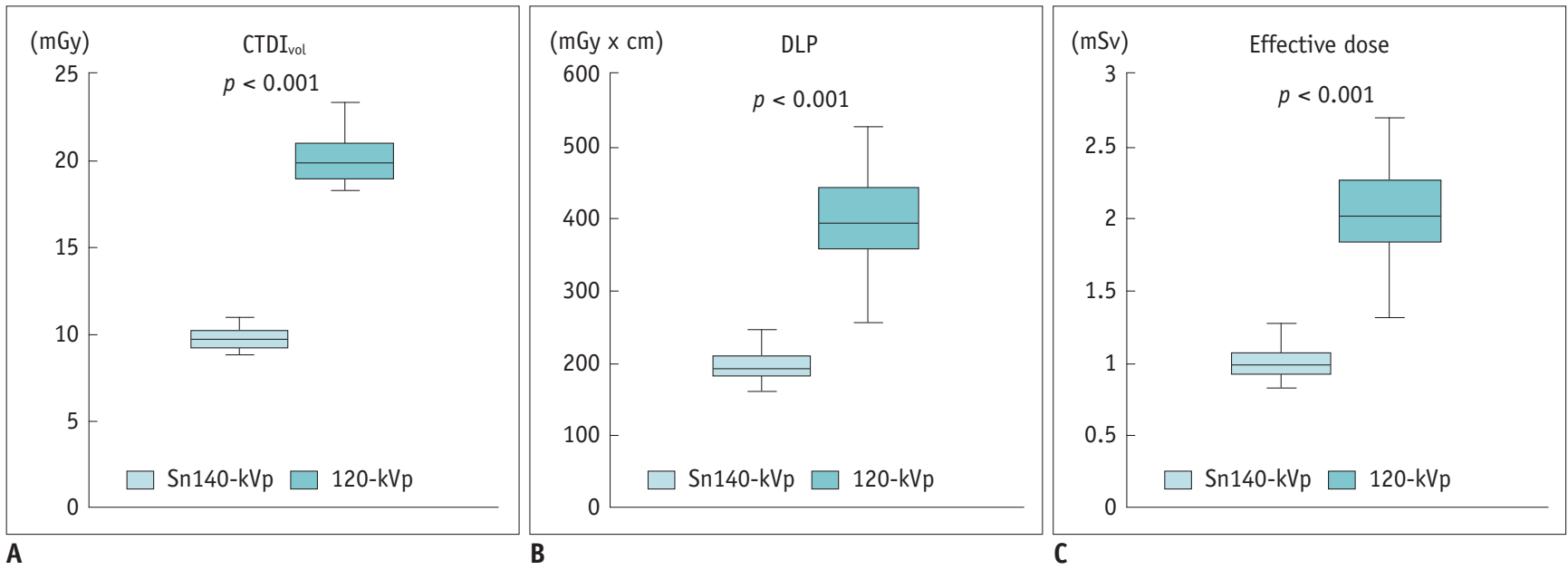

Fig. 7. Box-and-whiskers plots for radiation dose.

$\mathrm{CTDI}_{\text {vol }}(\mathbf{A})$, DLP (B), and effective dose (C) are significantly reduced in cervical spine CT with Sn140-kVp. CTDI $I_{\text {vol }}=$ volume CT dose index, DLP = dose length product

Table 8. Relationship between Body Habitus and Radiation Dose

\begin{tabular}{|c|c|c|c|c|c|c|c|c|}
\hline & \multicolumn{4}{|c|}{ Sn140-kVp } & \multicolumn{4}{|c|}{$120-k V p$} \\
\hline & \multicolumn{2}{|c|}{ Shoulder RL Diameter } & \multicolumn{2}{|c|}{ Neck AP Diameter } & \multicolumn{2}{|c|}{ Shoulder RL Diameter } & \multicolumn{2}{|c|}{ Neck AP Diameter } \\
\hline & Coefficient & $P$ & Coefficient & $P$ & Coefficient & $P$ & Coefficient & $P$ \\
\hline $\mathrm{CTDI}_{\text {vol }}$ & 0.366 & 0.043 & 0.366 & 0.005 & 0.620 & $<0.001$ & 0.641 & $<0.001$ \\
\hline DLP & 0.450 & 0.011 & 0.491 & $<0.001$ & 0.721 & $<0.001$ & 0.528 & $<0.001$ \\
\hline Effective dose & 0.450 & 0.011 & 0.491 & $<0.001$ & 0.716 & $<0.001$ & 0.512 & $<0.001$ \\
\hline
\end{tabular}


in the lower cervical spine region compared with the conventional CT with $120-\mathrm{kVp}$. The superior image quality in the lower cervical spinal area for Sn140-kVp acquisitions could be due to the utilization of a high tube voltage, an increased rate of the dose-efficient photons by the tin filter causing a decrease in the energy absorption by the patients and a decrease in the dark band and streak artifacts. The attenuation at the level of C3/4 was significantly lower in Sn140-kVp acquisitions than in the conventional acquisitions. However, it did not affect the intermodality agreement in the assessment of the disc morphology.

A previous study evaluated the intermodality agreement between MRI and CT without the application of the spectral shaping by a tin filter, for herniated discs (24). In that study, the intermodality agreement gradually decreased towards the lower cervical spine level, akin to ours. However, in our study, the intermodality agreement in the lower cervical spinal regions tended to be better in Sn140$k V p$ scans than that in the conventional scans.

Our study had limitations. First, it was a retrospective study. Thus, the included patients could not undergo both Sn140-kVp and conventional $120-k V p$ scans. However, the estimated body habitus and demographics that could affect the image quality and radiation dose were not significantly different between the two groups. The parameters between the two protocols were the same except for the use of tin filter, tube voltage, and reference tube currenttime products. Second, although information about the patients and protocols was completely removed from a picture archiving and communication system (PACS), we acknowledge that the readers could have recognized the protocols due to the mild difference in the appearance of the images. Third, the intermodality agreement between CT and MRI was assessed only by the disc morphology in spite of many pathologies of the cervical spine. We thought disc herniation was a suitable pathology for this assessment as it was one of the common abnormalities in patients with posterior neck discomfort, and the dark-band and streak artifacts were usually more severe within the central spinal canals. Fourth, although CT is a primary tool for evaluation of fracture, the presence of fractures was not determined. We thought that fracture could affect the image quality due to edema or hemorrhage associated with it and therefore, the cases with fractures were excluded from our study. Instead, the SNR and CNR of the cervical spinal body were calculated. Fifth, iterative reconstruction was not applied in this study. Previous studies demonstrated that iterative reconstruction offered a more noise reduction than filtered back-projection reconstruction (14, 25-27). Thus, a combination of iterative reconstruction and spectral shaping by a tin filter in the cervical spine CT would be more helpful to reduce the artifacts in the lower cervical region.

In conclusion, cervical spine CT with Sn140-kVp decreases artifacts in the lower cervical spinal region, while reducing the radiation exposure in patients, compared with the conventional $120-k V p$ CT. Thus, a cervical spine CT with Sn140-kVp can be helpful to overcome the artifacts in the lower cervical region.

\section{Conflicts of Interest}

The authors have no financial conflicts of interest.

\section{ORCID}

Hye Jung Choo

https://orcid.org/0000-0003-3941-6989

Su Min Lee

https://orcid.org/0000-0002-4616-5287

\section{REFERENCES}

1. Daffner RH, Hackney DB. ACR Appropriateness Criteria on suspected spine trauma. J Am Coll Radiol 2007;4:762-775

2. Douglas-Akinwande AC, Rydberg J, Shah MV, Phillips MD, Caldemeyer KS, Lurito JT, et al. Accuracy of contrast-enhanced MDCT and MRI for identifying the severity and cause of neural foraminal stenosis in cervical radiculopathy: a prospective study. AJR Am J Roentgenol 2010;194:55-61

3. Barrett JF, Keat N. Artifacts in CT: recognition and avoidance. Radiographics 2004;24:1679-1691

4. Mori I, Machida Y, Osanai M, Iinuma K. Photon starvation artifacts of $\mathrm{X}$-ray $\mathrm{CT}$ : their true cause and a solution. Radiol Phys Technol 2013;6:130-141

5. Mayo-Smith WW, Hara AK, Mahesh M, Sahani DV, Pavlicek W. How I do it: managing radiation dose in CT. Radiology 2014;273:657-672

6. Mueck FG, Roesch S, Geyer L, Scherr M, Seidenbusch M, Stahl $R$, et al. Emergency CT head and neck imaging: effects of swimmer's position on dose and image quality. Eur Radiol 2014;24:969-979

7. Primak AN, Ramirez Giraldo JC, Liu X, Yu L, McCollough $\mathrm{CH}$. Improved dual-energy material discrimination for dualsource CT by means of additional spectral filtration. Med Phys 2009;36:1359-1369

8. Braun FM, Johnson TR, Sommer WH, Thierfelder KM, Meinel FG. Chest CT using spectral filtration: radiation dose, image quality, and spectrum of clinical utility. Eur Radiol 2015;25:1598-1606 
9. Gordic S, Morsbach F, Schmidt B, Allmendinger T, Flohr T, Husarik D, et al. Ultralow-dose chest computed tomography for pulmonary nodule detection: first performance evaluation of single energy scanning with spectral shaping. Invest Radiol 2014; 49:465-473

10. Haubenreisser H, Meyer M, Sudarski S, Allmendinger T, Schoenberg S0, Henzler T. Unenhanced third-generation dualsource chest CT using a tin filter for spectral shaping at 100kVp. Eur J Radiol 2015;84:1608-1613

11. May MS, Brand M, Lell MM, Sedlmair M, Allmendinger T, Uder $\mathrm{M}$, et al. Radiation dose reduction in parasinus $\mathrm{CT}$ by spectral shaping. Neuroradiology 2017;59:169-176

12. Kim CR, Jeon JY. Radiation dose and image conspicuity comparison between conventional $120 \mathrm{kVp}$ and $150 \mathrm{kVp}$ with spectral beam shaping for temporal bone CT. Eur J Radiol 2018; 102:68-73

13. Suntharalingam S, Mikat C, Wetter A, Guberina N, Salem A, Heil $P$, et al. Whole-body ultra-low dose CT using spectral shaping for detection of osteolytic lesion in multiple myeloma. Eur Radiol 2018;28:2273-2280

14. Becce F, Ben Salah Y, Verdun FR, Vande Berg BC, Lecouvet FE, Meuli R, et al. Computed tomography of the cervical spine: comparison of image quality between a standard-dose and a low-dose protocol using filtered back-projection and iterative reconstruction. Skeletal Radiol 2013;42:937-945

15. Lee SH, Lee YH, Suh JS. Accelerating knee MR imaging: compressed sensing in isotropic three-dimensional fast spinecho sequence. Magn Reson Imaging 2018;46:90-97

16. Fardon DF, Williams AL, Dohring EJ, Murtagh FR, Gabriel Rothman SL, Sze GK. Lumbar disc nomenclature: version 2.0: recommendations of the combined task forces of the North American Spine Society, the American Society of Spine Radiology and the American Society of Neuroradiology. Spine J 2014;14:2525-2545

17. Deak PD, Smal Y, Kalender WA. Multisection CT protocols: sex- and age-specific conversion factors used to determine effective dose from dose-length product. Radiology 2010;257:158-166

18. Landis JR, Koch GG. The measurement of observer agreement for categorical data. Biometrics 1977;33:159-174

19. Krauss B, Grant KL, Schmidt BT, Flohr TG. The importance of spectral separation: an assessment of dual-energy spectral separation for quantitative ability and dose efficiency. Invest Radiol 2015;50:114-118

20. Stolzmann P, Leschka S, Scheffel H, Rentsch K, Baumüller S, Desbiolles $L$, et al. Characterization of urinary stones with dual-energy CT: improved differentiation using a tin filter. Invest Radiol 2010;45:1-6

21. Wang CK, Tsai JM, Chuang MT, Wang MT, Huang KY, Lin RM. Bone marrow edema in vertebral compression fractures: detection with dual-energy CT. Radiology 2013;269:525-533

22. Jeon JY, Lee SW, Jeong YM, Baek HJ. The effect of tube voltage combination on image artefact and radiation dose in dual-source dual-energy CT: comparison between conventional $80 / 140 \mathrm{kV}$ and 80/150 kV plus tin filter for gout protocol. Eur Radiol 2018 Jul 9 [Epub ahead of print]. https://doi. org/10.1007/s00330-018-5622-9

23. Wichmann JL, Hardie AD, Schoepf UJ, Felmly LM, Perry JD, Varga-Szemes A, et al. Single- and dual-energy CT of the abdomen: comparison of radiation dose and image quality of 2nd and 3rd generation dual-source CT. Eur Radiol 2017;27:642-650

24. Yi JS, Cha JG, Han JK, Kim HJ. Imaging of herniated discs of the cervical spine: inter-modality differences between 64-slice multidetector CT and 1.5-T MRI. Korean J Radiol 2015;16:881888

25. Iyama $Y$, Nakaura T, Iyama A, Kidoh M, Katahira K, Oda $S$, et al. Feasibility of iterative model reconstruction for unenhanced lumbar CT. Radiology 2017;284:153-160

26. Notohamiprodjo S, Stahl R, Braunagel M, Kazmierczak PM, Thierfelder KM, Treitl KM, et al. Diagnostic accuracy of contemporary multidetector computed tomography (MDCT) for the detection of lumbar disc herniation. Eur Radiol 2017; 27:3443-3451

27. Costello JE, Cecava ND, Tucker JE, Bau JL. CT radiation dose: current controversies and dose reduction strategies. AJR Am J Roentgenol 2013;201:1283-1290 